\title{
Dor na pancreatite crônica
}

\author{
Dulce Reis Guarita \\ Guilherme Eduardo Gonçalves Felga \\ Carlos de Barros Mott
}

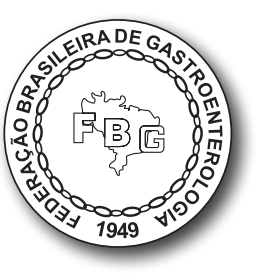

A dor é a principal queixa clínica dos pacientes com pancreatite crônica, comprometendo enormemente a qualidade de vida dos mesmos e deve ser avaliada com a utilização de instrumento específico (Pancreatitis Quality of Life Instrument - PanQOLI).

A suspensão da ingestão alcoólica e do tabagismo não impede que as lesões histológicas progridam, mas, por permitir menor componente inflamatório, pode diminuir o quadro álgico.

A dor pode ocorrer em surtos (tipo A) ou ser contínua (tipo B), deve ser avaliada por escala visual analógica (EVA) e ser tratada com a menor dose possível de medicação que a alivie.

O ponto mais importante para que seja corretamente abordada é a evidência de alterações em todos os níveis do sistema nervoso, central e periférico nestes pacientes, estando as alterações neuronais e os receptores PAR-2 e TRPV1 para a dor bem demonstrados. Estas modificações só ocorrem em portadores de pancreatite crônica e de carcinoma de pâncreas.

Diante da existência desse comprometimento que vai além da glândula pancreática, pacientes submetidos a derivações ou mesmo a ressecções pancreáticas podem apresentar dor, má resposta a bloqueios celíacos e alterações eletroencefalográficas, e os doentes podem responder à pré-gabalina:

Nas crises de agudização (crises dolorosas), a simples restrição da alimentação, por 1 ou 2 dias, ou mesmo a manutenção da dieta à base de hidratos de carbono, associada à administração de analgésicos injetáveis, é suficiente para o retorno às condições normais em curto período. A utilização da sonda nasogástrica se justifica apenas quando as crises são muito intensas e, sobretudo, quando acompanhadas de vômitos repetidos. 
A analgesia deve seguir os princípios de pain reliefladder, preconizados pela Organização Mundial da Saúde, iniciando-se com o paracetamol, não havendo consenso sobre a utilização de anti-inflamatórios não esteroides (AINEs). O cloridrato de tramadol (50 a $100 \mathrm{mg}$ em até a cada 6 horas, em injeção intravenosa lenta) ou o cloridrato de buprenorfina $(0,3 \mathrm{mg}$, por via intramuscular, em até a cada 6 horas) são opções para esses pacientes, aliando boa potência analgésica a poucos efeitos adversos. Se houver persistência da manifestação dolorosa após 1 semana, apesar dos cuidados médicos, complicações podem ter se instalado, dentre as quais se destacam a necrose pancreática e os pseudocistos. O cloridrato de tramadol tem efeitos semelhantes aos da morfina, com menos efeitos colaterais.

A pré-gabalina por via oral (75 a $300 \mathrm{mg} / \mathrm{dia})$ pode melhorar a dor e, se necessário, os tricíclicos podem ser associados.

Os antioxidantes necessitam de mais estudos controlados para que sua eficácia possa ser avaliada.

Apesar das controvérsias na literatura, a reposição enzimática semelhante à utilizada em insuficiência exócrina pode levar à redução da frequência e da intensidade das crises dolorosas por um mecanismo de feedback negativo na regulação da secreção pancreática.

Até o momento, não há justificativas para o uso de octreotide e outras propostas (estimulação magnética transcraniana, acupuntura, ervas medicinais, homeopatia), lembrando que placebos podem atuar em 20 a 30\% dos pacientes por determinado período.

\section{TRATAMENTO ENDOSCÓPICO}

Vários procedimentos endoscópicos têm sido propostos no tratamento das pancreatites crônicas, sobretudo em crises dolorosas recidivantes, colestase, pseudocistos e derrames cavitários. São preconizados esfincterotomia pancreática, isolada ou associada à retirada de cálculos localizados na cabeça do pâncreas, com litotripsia, colocação de endopróteses no ducto pancreático principal ou na via biliar, drenagem de cistos de retenção e/ou pseudocistos e alcoolização de plexo celíaco, guiada por ecoendoscopia.

A endoterapia, associada à litotripsia extracorpórea, seria uma "ponte" capaz de postergar a indicação cirúrgica. Assim, quando utilizada em portadores de estenoses, deixa $100 \%$ dos pacientes sem dor logo após o procedimento, $50 \%$ sem dor após 5 anos e 30\% farão cirurgia posteriormente. Já a endoscopia em litíase tem como resultados a necessidade de fragmentação em $75 \%$ dos casos, $90 \%$ dos pacientes ficam sem dor logo após o procedimento, 50\% sem dor após 5 anos e $30 \%$ farão cirurgia posteriormente. 
A pancreatoscopia peroral, associada à litotripsia intracorpórea, é opção terapêutica promissora e necessita de maior número de pacientes tratados e estudos controlados adequados para que seja validada.

A infiltração do plexo celíaco com corticosteroides não é procedimento isento de complicações, como abscessos, diarreia, paraplegia, aumento da dor e hipotensão ortostática, e deve ser proposta com cautela e para casos específicos.

\section{TRATAMENTO CIRÚRGICO}

Cerca de $20 \%$ dos pacientes portadores de pancreatite crônica são tratados cirurgicamente, especialmente quando há persistência das manifestações dolorosas, ou quando surgem complicações durante a evolução da doença, como pseudocistos pancreáticos complicados, ascite, derrame pleural, necrose infectada, abscessos, fístulas pancreáticas, hemorragia digestiva de repetição ou incoercível e icterícia persistente (Tabela 1).

\begin{tabular}{lcc} 
Tabela 1 & Indicações de tratamento cirúrgico em pancreatite crônica - HC-FMUSP \\
Indicação & $n$ & $\%$ \\
Dor persistente & 110 & 54 \\
\hline Pseudocisto & 20 & 9 \\
\hline Ascite & 19 & 8,6 \\
\hline Icterícia & 16 & 7,2 \\
\hline Abscesso & 13 & 5,9 \\
\hline Derrame pleural & 8 & 3,6 \\
\hline Hemorragia digestiva & 3 & 1,3 \\
\hline Fístula & 2 & 0,9 \\
\hline Associação de fatores & 2 & 0,9 \\
\hline Total & 193 & 100 \\
\hline HC-FMUSP: Hospital das Clínicas da Faculdade de Medicina da Universidade de São Paulo. Fonte: Cunha \\
et al.,20046
\end{tabular}

O tratamento cirúrgico deve controlar a dor, corrigir a obstrução canalicular, fazer ressecções econômicas do parênquima glandular, retornar as enzimas ao tubo digestivo e corrigir as alterações de órgãos e estruturas vizinhas. De modo geral, as intervenções cirúrgicas se dividem em dois grandes grupos, as derivações e as ressecções, sendo preferíveis as primeiras em relação às últimas, por permitirem maior preservação do parênquima pancreático.

Em revisão feita (Cochrane Database) tanto sobre a pancreatite crônica com dilatação ductal quanto sobre as fases iniciais da afecção, o procedimento 
cirúrgico foi superior ao endoscópico, tanto para o controle da dor, quanto para melhorar a função pancreática.

A melhor evolução do paciente com pancreatite crônica é obtida se a cirurgia for indicada até três anos após o início dos sintomas dolorosos, sendo a instalação de dor de origem central uma preocupação quando a cirurgia é adiada. A probabilidade de melhora da dor após o procedimento cirúrgico se baseia no tempo de duração do quadro álgico, no uso de opioides antes da cirurgia e no número de procedimentos endoscópicos pré-operatórios.

Outra abordagem terapêutica cirúrgica proposta mais recentemente é a pancreatectomia total com autotransplante de ilhotas (TPIAT), sendo que sua utilização requer indicação precisa e que o paciente não tenha condições para as cirurgias de derivação pancreática em uso há vários anos, com bons resultados.

\section{CONCLUSÕES}

A dor é o sintoma mais comum e de mais difícil abordagem em pacientes portadores de pancreatite crônica, levando a maior morbidade e a importante comprometimento da qualidade de vida dos mesmos.

O tratamento do quadro álgico deve ser feito passo a passo, com a suspensão do álcool e do tabagismo, a adoção de dieta pobre em gorduras (mas não sem elas) e a reposição de enzimas pancreáticas (controversa na literatura). Utiliza-se, inicialmente, paracetamol, com ou sem AINEs (estes últimos também de uso controverso), prosseguindo, se necessário, com narcóticos de menor e, se ineficazes, maior eficácia.

Neuromoduladores, como a pré-gabalina, podem ser usados e o resultado obtido é bom.

O tratamento endoscópico eficaz se associa à litotripsia extracorpórea e, recentemente, à litotripsia intracorpórea com pancreatoscopia; no entanto, até o momento, seria uma "ponte" para o tratamento cirúrgico de dor persistente em pancreatite crônica.

As cirurgias, desde que possível, devem preservar o pâncreas e seu sucesso se relaciona ao tempo entre o aparecimento da dor e a indicação cirúrgica, ao uso prévio de opioides e ao número de procedimentos endoscópicos precedendo o ato operatório.

Finalmente, em casos específicos, a pancreatectomia total com autotransplante de ilhotas surge como alternativa para tratar dor persistente. 


\section{REFERÊNCIAS BIBLIOGRÁFICAS}

1. Anderson MA, et al. Mechanism, assessment and management of pain in chronic pancreatitis: recommendations of a multidisciplinary study group. Pancreatology. 2016;16(1):83-94.

2. Ahmed AU, et al. Endoscopic or surgical intervention for painful obstructive chronic pancreatitis. Cochrane Database Syst Rev. 2012;1.

3. Bellin MD, et al. Total pancreatectomy and islet autotransplantation in chronic pancreatitis: recommendations from pancreas fest. Pancreatology. 2014;14(1):27-35.

4. Beyna T, Gerges C, Neuhaus H. Endoscopic treatment of pancreatic duct stones under direct vision. Revolution or resignation? A systematic review. Dig Endosc. 2017.

5. Cahen DL, et al. Long-term outcomes of endoscopic vs surgical drainage of the pancreatic duct in patients with chronic pancreatitis. Gastroenterology. 2011;141(5):1690-5.

6. Cunha JEM, et al. Surgical and interventional treatment of chronic pancreatitis. Pancreatology. 2004;4:540-50.

7. Frøkjær JB, et al. Reduced cortical thickness of brain areas involved in pain processing in patients with chronic pancreatitis. Clin Gastroenterol Hepatol. 2012:10:434-8

8. Löhr JM, et al. United European Gastroenterology evidence-based guidelines for the diagnosis and therapy of chronic pancreatitis (HaPanEU). United European Gastroenterol J. 2017;5(2):153-99.

9. Olesen SS, et al. Pregabalin reduces pain in patients with chronic pancreatitis in a randomized, controlled trial. Gastroenterology. 2011;141(2):536-43.

10. Rupasinghe SN, Siriwardena AK. Long-term outcome of patients with chronic pancreatitis treated with micronutrient antioxidant therapy. Hepatobiliary Pancreat Dis Int. 2017;16(2):209-14. 